\title{
OBSTRUCTED LABOUR IN PREGNANCY WITH UTERUS DIDELPHYS: A RARE CASE REPORT
}

\author{
V. Gomathi ${ }^{1}$, K. Mythili2 ${ }^{2}$ Sridevi $^{3}$
}

\section{HOW TO CITE THIS ARTICLE:}

V. Gomathi, K. Mythili, Sridevi. "Obstructed Labour in Pregnancy with Uterus Didelphys: A Rare Case Report". Journal of Evolution of Medical and Dental Sciences 2015; Vol. 4, Issue 43, May 28; Page: 7563-7566, DOI: $10.14260 /$ jemds/2015/1098

ABSTRACT: Uterine malformations are congenital anomalies of female genital tract caused by fusion or resorption defects during embryogenesis. Uterus didelphys is one of the congenital uterine malformation which is rare and sometimes not even diagnosed. We report a rare case of 20 years old lady with uterus didelphys with single successful pregnancy in left hemi uterus presented to us in obstructed labour. A live full term male baby of weight of 3200 grams was extracted from left hemi uterus by lower segment cesarean section. On right side, there was a non-pregnant uterus which was lying posterior to left pregnant uterus, each hemi uterus is attached to one fallopian tube and one overy. She also has a longitudinal vaginal septum.

KEYWORDS: Uterus didelphys, uterine malformations, vaginal septum, lower segment cesarean section.

INTRODUCTION: The human uterus is of paramesonephric in origin. During embryogenesis fusion of two mullerian ducts and establishment of vaginal canal are usually completed between 10 and $17^{\text {th }}$ week of intrauterine life. Any degree of failure of fusion of mullerian ducts or subsequent failure of resorption of tissues results in spectrum of clinical malformations. Uterus didelphys is a condition of failure of lateral fusion results in two hemi uterus and cervices. According to American fertility society classification of utero vaginal anomalies, uterus didelphys belongs to class 3 . It is a lateral fusion defect of mullerian ducts with symmetrical unobstructed didelphic uterus having complete vaginal septum. The cause of fusion failure is not known. It is a rare uterine anomaly and according to one estimate, it occurs in $0.1 \%-0.5 \%$ healthy fertile population. ${ }^{1}$ Low incidence of uterus didelphys is reflected in literature by paucity of data regarding reproductive performances. Of all the uterine anomalies, didelphic uterus is associated with successful pregnancy. Uterus didelphys is associated with developmental urinary tract anomalies about 20-30\% and cervical incompetence also. ${ }^{2}$

These patients usually have no difficulties in menstruation and coitus except when there is a midline longitudinal vaginal septum. Occasionally one side may be obstructed by transverse vaginal septum and have dysmenorrheal and hematocolpus. It is associated with various obstetric complications such as spontaneous abortion, preterm labour, cervical incompetence, malpresentations and obstructed labour.

CASE REPORT: A 20 years old unregistered primi gravida presented to our hospital with 39 weeks of gestation in labour, referred from PHC for safe confinement. She had regular menstrual cycles with no dysmenorrhoea. No significant past and family history. Her vitals were normal. On examination uterus was term and acting with single fetus in cephalic presentation with good fetal heart rate. Longitudinal vaginal septum was seen on per speculam examination (Fig. 1). On vaginal examination there were two cervices, one on each side of vaginal septum was found. Left cervix was dilated to 3-4 cms, fully effaced, vertex at -1 station with large caput and absent membranes. 
All her routine blood and urine investigations were normal. Emergency LSCS was done in a usual way for obstructed labour and extracted an alive, full term, male baby of weight $3.2 \mathrm{~kg}$. Intraoperatively we observed a non-pregnant uterus of about 10 weeks size found in the pelvis, lying posterior and right of the pregnant left hemi uterus. There was a intervening thick fibrous band in between both hemi uterus, extending between bladder and rectum. One fallopian tube and one ovary were attached to each hemi uterus (Fig. 2). She had primary atonic PPH, which was managed by oxytocics and blood transfusion. She had uneventful postoperative period. Mother and baby were discharged on $8^{\text {th }}$ Post-operative day.

DISCUSSION: Congenital defects of reproductive tract are often associated with great liability for preterm labour, abnormal presentations, dystocia and increased necessity for cesarean section. These women require meticulous prenatal care. Non obstructive type uterus didelphys is usually asymptomatic. Workup should include 3D USG, HSG, MRI and IVP to conform or exclude associated urinary tract anomalies. 3D ultrasound offers $100 \%$ specificity and MRI is the most sensitive imaging modality in diagnosing uterine anomalies. ${ }^{3}$ A specific association of uterus didelphys is obstructed unilateral vagina resulting in hematocolpus and ipsilateral renal and ureter agenesis known as Wunderlich Herlyn Werner syndrome, a rare but well established anomaly. ${ }^{4}$ Our patient did not show any urinary tract anomalies in MRI which was done after 3 months after delivery.

Simultaneous pregnancies in each hemi uterus of didelphic uterus can occur rarely. These twins are always dizygotic. This is supported by reports in which second twin was delivered after a long interval ranging from 3 hours, 5 days to 8 weeks after delivery of Ist twin.5,6

Management of delivery is contravercial. Some believe that vaginal delivery can be safely achieved, others advocate cesarean section. Ravesia et al. ${ }^{7}$ Reported a 8\% incidence of uterine rupture $(2 / 25)$ in women with congenitally malformed uteri compared with $0.61 \%(11 / 1788)$ in the normal uteri $(\mathrm{p}=0.013)$ who were attempting VBAC.

Reproductive performances of 49 women with uterus didelphys were evaluated by Heinonen et $\mathrm{al}^{8}$ in 2009 and observed miscarriage $20 \%$, cesarean section $22 \%$, prematurity $24 \%$, malpresentation $20 \%$ and perinatal mortality 5.3\%. Poor reproductive outcomes are thought to be due to diminished uterine volume and decreased perfusion in each hemi uterus. The walls of the abnormal uteri tend to become abnormally thin as the pregnancy advance and thickness can be inconsistent over different aspects of myometrium.

In case of single pregnancy in uterus didelphys, literature shows the right hemi uterus having pregnancy predominantly.1,2,9 In uterus didelphys, non-pregnant hemi uterus is also subjected to hormonal influences as the pregnant hemi uterus and it remains as a pelvic organ posterior to the pregnant hemi uterus ${ }^{10}$ and hampers delivery of the fetus. In our case, we report a single successful pregnancy in left hemi uterus which is very rare.

CONCLUSION: Interestingly in our case report, this woman had single successful pregnancy in the left hemi uterus and gave birth to a boy baby by cesarean section. However the mother did not have the history of dysmenorrheal, abortions, preterm delivery. Conclusively we also state that patients with uterus didelphys belong to high risk group and must need meticulous prenatal care. Therefore it is of great importance for clinicians to detect these abnormalities of reproductive tract in earlier by imaging modalities. 


\section{CASE REPORT}

The prompt, accurate diagnosis of congenital uterine malformations and appropriate surgical interventions are essential to prevent complications, and to provide the best possible pregnancy outcome for all such patient.

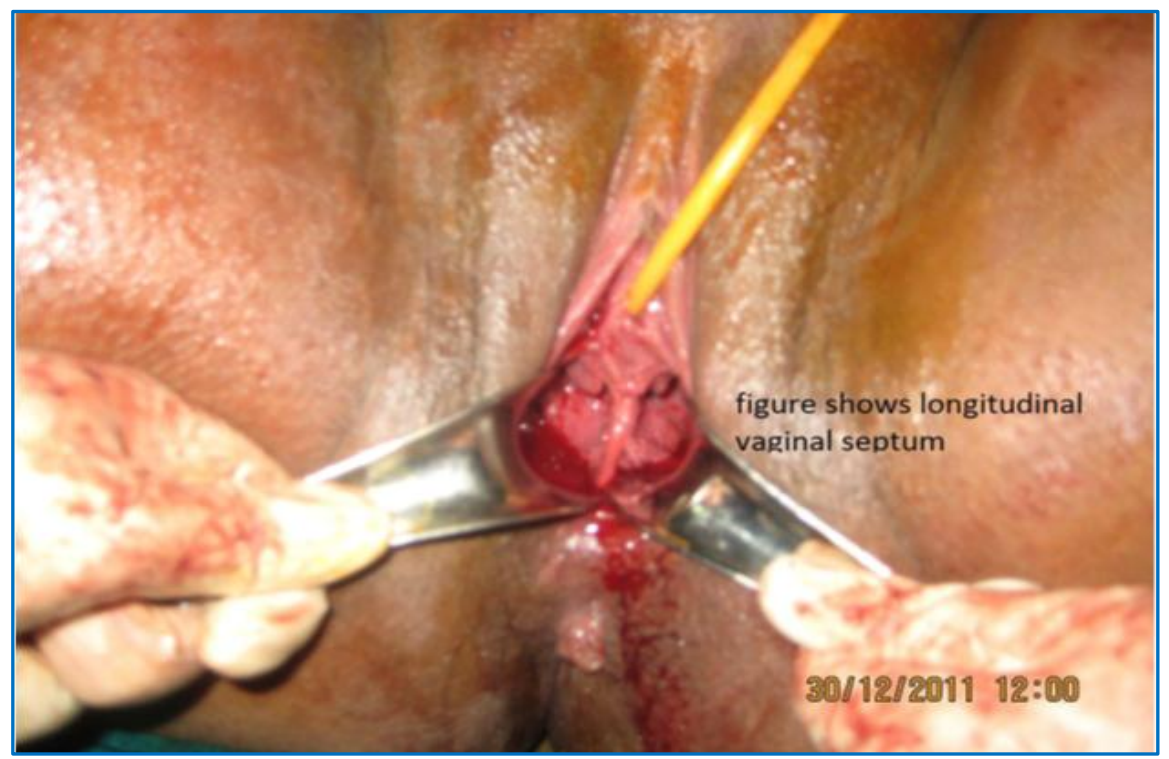

Fig. 1: Shows longitudinal vaginal septum

Fig. 2: Shows non- pregnant right uterus with pregnant left uterus after cesarean section with a fold of peritoneum separating both uterus. Each hemi uterus has its one fallopian tube and one ovary.

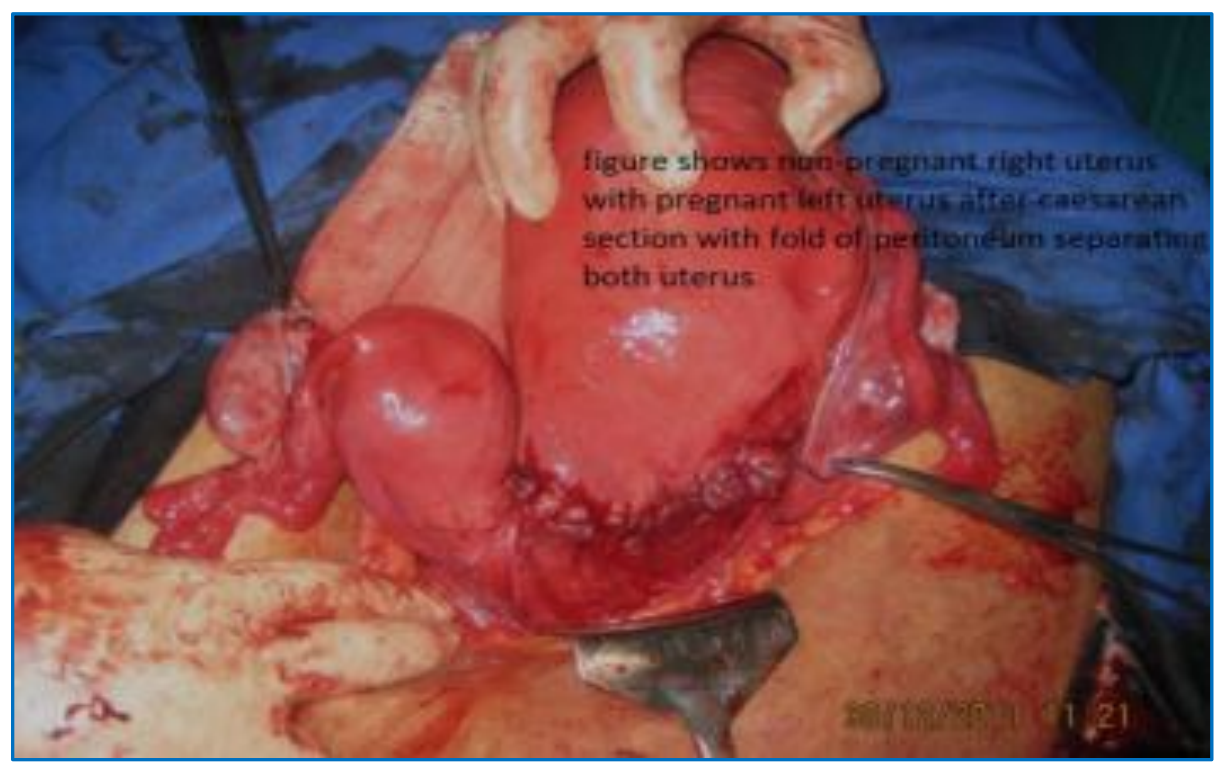

Fig. 2 


\section{REFERENCES:}

1. Mohd Sohail, Hina Khan et al. Uterus didelphys having single pregnancy in right hemi uterus; A case report. J Chin clin medicine 2010; 5; 46-49.

2. Green LK, Harris RE. Uterine anomalies; frequency of diagnosis and associated obstetrical complications. Obstet Gynecol 1976; 47: 427-429.

3. $\mathrm{Wu} \mathrm{MH}$, Huang Ke, Detection of congenital mullerian duct anomalies using 3D USG. J Clin ultrasound. 1997; 25(9)487-492.

4. Purandare $\mathrm{CN}$ et al. Hematometra in uterus with didelphys with obstructed right hemi vagina with right renal agenesis. J Obstet Gynecol India 2011, 61(2); 210-212.

5. Bhattacharys S, Misti PK. Twin pregnancy in a women with uterus didelphys. Online J health Alliedscs. 2010; 9(4)24.

6. Nohara M, Nakayama. Twin pregnancy in each uterus didelphys with a delivery interval of 66 days.Br. J Obstet Gynecol 2003; 110(3); 331-332.

7. Ravasia DJ, Brain PH. Incidence of uterine rupture among women with mullerian duct anomalies who attempt vaginal birth after C.S. Am. J Obstet Gynecol Oct 1999; 181(4); 877-81.

8. Heinonen P.K. Clinical implications of didelphic uterus - lonf term follow up of 49 cases. Eur. J Obstet Gynecol Reprod Biol. 2000; 91(2); 183-190.

9. Gomathy E, Sheela SR. Urerus didelphys with unilateral vaginal obstruction having single pregnancy in her right horn. J Clin Biomed Sci. 2013; 3(3); 143-145.

10. Eds-Speroff L and Friet Z. The uterus. In clinical Gynecological endocrinology and infertility. $7^{\text {th }}$ edition.2005; p.132.

\section{AUTHORS:}

1. V. Gomathi

2. K. Mythili

3. Sridevi

\section{PARTICULARS OF CONTRIBUTORS:}

1. Assistant Professor, Department of Obstetrics and Gynaecology, Melmaruvathur Adiparasakthi Institute of Medical Science and Research.

2. Associate Professor, Department of Obstetrics and Gynaecology, Melmaruvathur Adiparasakthi Institute of Medical Science and Research.

FINANCIAL OR OTHER

COMPETING INTERESTS: None
3. Professor, Department of Obstetrics and Gynaecology, ESI PGIMSR, K. K. Nagar, Chennai.

\section{NAME ADDRESS EMAIL ID OF THE CORRESPONDING AUTHOR:}

Dr. V. Gomathi, Assistant Professor, Department of Obstetrics and Gynaecology, Melmaruvathur Adiparasakthi Institute of Medical Science and Research.

E-mail:drvgomathi@gmail.com

Date of Submission: 03/05/2015. Date of Peer Review: 04/05/2015. Date of Acceptance: 20/05/2015. Date of Publishing: 28/05/2015. 